\title{
Characteristic lengths and maximum entropy estimation from probe signals in the ellipsoidal bubble regime
}

\author{
D. Santana $^{\mathrm{a}, *}$, J. Rodríguez-Rodríguez ${ }^{\text {b }}$, J.A. Almendros-Ibáñez ${ }^{\mathrm{a}}$, \\ C. Martínez-Bazán ${ }^{\mathrm{c}}$ \\ ${ }^{a}$ Department of Thermal and Fluids Engineering, Universidad Carlos III de Madrid, Avda. de la Universidad 30, \\ 28911 Leganés, Madrid, Spain \\ ${ }^{\mathrm{b}}$ Department of Mechanical and Aerospace Engineering, University of California, 9500 Gilman Drive, La Jolla, San Diego, \\ CA 92093 0411, USA

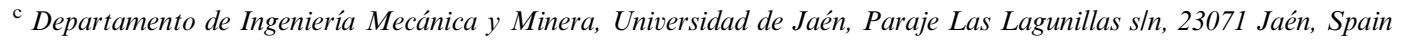

\begin{abstract}
The bubble size, surface and volume distributions in two and three phase flows are essential to determine energy and mass transfer processes. The traditional approaches commonly use a conditional probability density function of chord lengths to calculate the bubble size distribution, when the bubble size, shape and velocity are known. However, the approach used in this paper obtains the above distributions from statistical relations, requiring only the moments inferred from the measurements given by a sampling probe. Using image analysis of bubbles injected in a water tank, and placing an ideal probe on the image, a sample of bubble diameter, shape factor and velocity angle are obtained. The samples of the bubble chord length are synthetically generated from these variables. Thus, we propose a semi parametric approach based on the maximum entropy (MaxEnt) distribution estimation subjected to a number of moment constraints avoiding the use of the complex backward transformation. Therefore, the method allows us to obtain the distributions in close form. The probability density functions of the most important length scales $\left(D, D_{20}, D_{30}, D_{32}\right)$, obtained applying the semi parametric approach proposed here in the ellipsoidal bubble regime, are compared with experimental measurements.
\end{abstract}

Keywords: Bubble size distribution; Chord lengths, Maximum entropy distribution; Characteristic length estimation

\section{Introduction}

The precise knowledge of bubble size distribution in two and/or three phase flows is an important aspect in many practical applications where the processes of energy and mass transfer are controlled by such distribution. Intrusive crossing probes were the first experimental techniques used, and in many instances the only

\footnotetext{
* Corresponding author. Tel.: +3491624 8462; fax: +34916249430.

E mail address: dsantana@ing.uc3m.es (D. Santana).
} 
ones available, especially in flows whose local void fraction may be larger than $20 \%$, as well as in 3D fluidized beds. In this latter case the opacity of the bed precludes the use of non-intrusive techniques.

Several phase-detection probes have been developed in the past, a detailed review of intrusive and non-intrusive measurement techniques applied to the field of fluidized beds, together with their advantages and disadvantages can be found in Cheremisinoff (1986). More recently Boyer et al. (2002) reviewed the application of these techniques to the case of two (gas/liquid) and three phase flows (gas/liquid/solid).

If the two-phase flow is one-dimensional, a two-point probe can be used to extract the chord-length and the bubble velocity from the signal. However, in a three-dimensional flow a four-point probe must be used to obtain reliable information. Furthermore, if the bubbles are in the elliptical regime, the angle of attack of the bubble with respect to the probe (Lucas and Mishra, 2005), the bubble shape (Luther et al., 2004; Guet et al., 2005), the bubble velocity and the time taken to cross the probe can be obtained when the bubble crosses the four-point probe (Mudde and Saito, 2001).

The analysis of the signal given by the phase detection probes is a very complex, and time-consuming procedure (Cartellier, 1999). Therefore, it is important to develop efficient techniques to obtain the bubble size, area or volume PDFs, as well as the mean variables, with the minimum number of samples possible. Once the bubble shape, angle of attack and chord-length distributions are established, three approaches are commonly used to obtain the bubble size distribution, namely parametric, semi-parametric and non-parametric. Lee et al. (1990), Liu and Clark (1995) and Clark et al. (1996) proposed parametric methods where a chord-length PDF is a priori estimated from the measurements (typically Gamma, Rayleigh or lognormal distributions). On the other hand, the bubble size distribution can be inferred by a backward transformation (Herringe and Davis, 1976; Liu and Clark, 1995; Clark et al., 1996) or by an optimization problem (Lee et al., 1990). Although these methods require the use of complicated backward transformations and are quite sensitive to discontinuities on the chord distribution, their main advantage is that they provide a bubble size distribution in closed form. However, for a large variety of distributions, the shape of this function is usually unknown and the estimate of the PDF parameters is not fully established.

In the case of non-parametric methods, the chord-length PDF does not need to be previously established. Dias et al. (2000) proposed a non-aprioristic statistical method for spherical bubbles to describe the influence of the angle of attack on the apparent (measured) and actual (real) distribution and they applied the model to their experiments, carried out in an air/water bubbly flow regime. Furthermore, Clark and Turton (1988) and Turton and Clark (1989) proposed a non-parametric numerical method, which was shown to be numerically unstable for small sampling records. Later on, Liu et al. (1996, 1998), using a Parzen window with ellipsoidal bubbles, proposed a non-parametric model that Santana and Macías-Machín (2000) extended to consider bubbles with non-zero angles of attack. Unfortunately, these methods incorporate the disadvantages that the bubble size distribution inferred is not in a closed form and the data must be divided in bins to take into account the bubble shape and/or velocity variation. On the other hand, the main advantage is that the models do not require the assumption of a given shape for the distribution.

Finally, the semi-parametric method proposed here, and based on the estimate of the maximum entropy density, hereafter denoted MaxEnt, subjected to a certain number of moment constraints (Ryu, 1993), avoids the disadvantages described above. Although, the PDF obtained should match a certain number of moments obtained from experimental measurements, the number of moments required is not predetermined. Therefore, although the problem reduces to fix the values of a few parameters, the number of parameters is a priori unknown.

To establish the size, the surface or the volume moments from the signals obtained by the sampling probes, these moments must be appropriately related to the moments of functions that only depend on the chordlength, the bubble shape or the angle of attack, since these variables can be measured or estimated from the measurements. Liu and Clark (1995) showed these relationships for the first two moments, both for an ellipsoidal and a truncated ellipsoidal bubble with pure vertical rising velocity. However, in this paper, the size, surface and volume moments calculated from the probe signal are obtained considering ellipsoidal bubbles raising with any angle of attack, and using the maximum entropy estimate of PDF to determine the number moments needed. As a result, we will be able to obtain the bubble size, surface or volume distributions.

As already mentioned above, the method proposed here is restricted to the ellipsoidal bubble regime reported by Clift et al. (1978) who dividing the bubble shapes in ellipsoidal, ellipsoidal cap and skirted, 
depending on the Reynolds and the Eötvös numbers with the Morton number as parameter, in two-phase (gas/liquid) systems. Other expressions have been proposed to take into account the contribution of the bubble shape to correlate the bubble size with the bubble velocity in gas/liquid reactors (Karamanev, 1994; Nguyen, 1998), which can be used to estimate the bubble shape from the probe signal.

In summary, in this paper we aim at estimating the bubble size, area and volume distributions from probe signals without assuming a given shape factor of ellipsoidal bubbles, minimizing the number of samples required to obtain reliable PDFs and avoiding the backward transformation procedure.

Although it is not the purpose of the present paper to discuss the measurement errors in chord-length determination from probe readings due to bubble probe interaction (Julia et al., 2005), uncertainly in bubble orientation and shape, etc., it is important to mention that significant errors in PDFs estimation arise in practice since the actual chord obtained from an ideal probe does not coincide with the measured chord, especially in cases of small chords (Dias et al., 2000) and distorted bubbles (Chaumat et al., 2005).

The paper is organized as follows: the second section is dedicated to obtain the maximum entropy (MaxEnt) distribution, subjected to an appropriate number of constrains on the raw data moments, with a stable and fast procedure. The third section introduces a simulation procedure and the implementation of the MaxEnt distribution estimate method as well as the experimental set-up. Finally a discussion of the results provided by the simulations, examining the errors generated when the bubble size distribution is estimated, is given in the last section.

\section{Raw moments and MaxEnt estimation}

\subsection{Raw moment estimators}

To estimate the raw moments (moments of the distribution referred to zero) we will consider the oblate, ellipsoidal bubble displayed in Fig. 1. This figure also shows the coordinate system, the variables used to determine the PDFs as well as their geometric relationships, where $D$ is the diameter of the bubble, $y$ is the chordlength, $v$ is the bubble velocity, $\theta$ is the zenith angle with respect to the coordinate system located at the bubble axis and $\alpha$ is a shape factor defined as the minor-to-major axis ratio.

The chord-length density function can be obtained assuming that the minimum distance between the center of the bubble and the probe path is uniformly distributed (Werther, 1974). Furthermore, Santana and MacíasMachín (2000) showed that, knowing the velocity vector, shape factor and the bubble diameter, the conditional probability density function of the chord-length measured by the probe can be given by

$$
P(y \mid D, \theta, \alpha)=\frac{2 y t^{2}}{D^{2} \alpha^{2}} ; \quad y \in\left(0, \frac{D \alpha}{t}\right) ; \quad \text { where } t^{2}=\frac{(\alpha \cdot \tan \theta)^{2}+1}{\tan ^{2} \theta+1}
$$

It can be observed from Eq. (1) that the raw moments of the bubble diameter can be related with the measurable variables. For example, applying the total probability theorem to the following expectation value:

$$
\frac{k+2}{2}\left\langle\left(\frac{y t}{\alpha}\right)^{k} \mid \theta, \alpha\right\rangle=\frac{k+2}{2} \int_{0}^{\infty}\left(\frac{y t}{\alpha}\right)^{k} P(y \mid \theta, \alpha) \mathrm{d} y=\frac{k+2}{2} \int_{0}^{\infty} \int_{\frac{y t}{\alpha}}^{\infty}\left(\frac{y t}{\alpha}\right)^{k} \frac{2 y t^{2}}{D^{2} \alpha^{2}} P(D \mid \theta, \alpha) \mathrm{d} D \mathrm{~d} y
$$

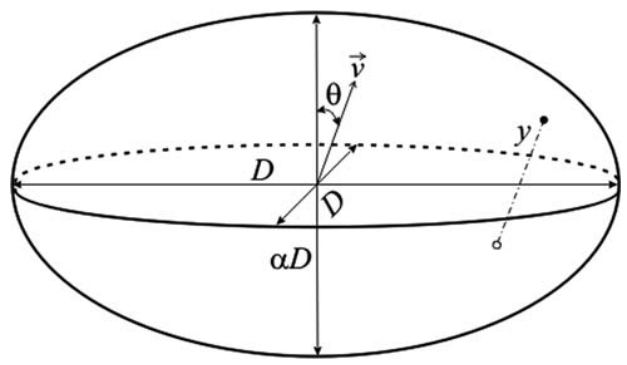

Fig. 1. Oblate spheroidal bubble model. 
and inverting the integral limits as

$$
\int_{0}^{\infty} \int_{\frac{y t}{\alpha}}^{\infty}\left(\frac{y t}{\alpha}\right)^{k} \frac{2 y t^{2}}{D^{2} \alpha^{2}} P(D \mid \theta, \alpha) \mathrm{d} D \mathrm{~d} y=\int_{0}^{\infty} \int_{0}^{\frac{D \alpha}{t}}\left(\frac{y t}{\alpha}\right)^{k} \frac{2 y t^{2}}{D^{2} \alpha^{2}} P(D \mid \theta, \alpha) \mathrm{d} y \mathrm{~d} D
$$

the diameter raw moments can be expressed as

$$
\left\langle D^{k} \mid \theta, \alpha\right\rangle=\frac{k+2}{2}\left\langle\left(\frac{y t}{\alpha}\right)^{k} \mid \theta, \alpha\right\rangle
$$

Finally using the following expectation function property:

$$
\langle h(X) g(Y)\rangle_{X, Y}=\left\langle h(X)\langle g(Y) \mid X=x\rangle_{Y}\right\rangle_{X}
$$

the $k$ th moment of the diameter can be related to the chord-length, the bubble shape and the incident angle as

$$
\frac{k+2}{2}\left\langle\left(\frac{y t}{\alpha}\right)^{k}\right\rangle=\left\langle D^{k}\right\rangle
$$

Thus, the different moments of the bubble diameter can be estimated from the chord-length, the angle of attack and the bubble shape as

$$
m_{k}(D)=\frac{k+2}{2 N} \sum_{i}^{N}\left(\frac{y_{i} t_{i}}{\alpha_{i}}\right)^{k}
$$

where $N$ is the number of samples taken. This estimation is in agreement with the experimental results, using a dual-sensor resistivity probe, carried out by Kalkach-Navarro et al. (1993), who obtained that, for nearly spherical bubbles, the mean diameter is 1.5 times the mean chord-length. Notice that Eq. (7) with $\alpha \quad 1$ and $k \quad 1$ recovers the same relationship between the mean diameter and the mean chordlength.

In practical cases, when intrusive probes are used to infer the bubble diameter distributions in two-phase flows, the variables obtained from probe signals depend on the number of points of the sampling probe. For two-point probes the variables extracted from the probe signal are the bubble residence time and the elapsed time between probes tips interception. From these variables, and knowing the distance between the two tips, the apparent bubble velocity and apparent chord-length can be determined. Furthermore, with the chord-length and bubble velocity obtained, the bubble shape factor of the detected bubble can be bounded if a suitable correlation between size and aspect ratio is employed. For example, in an almost stagnant medium, the bubble deformation is mainly a function of the Weber number (Taylor and Acrivos, 1964) or the Eötvös number (Wellek et al., 1966). Thus the shape factor is bounded between the Weber (or Eötvös) number based on the chord-length and bubble velocity obtained and that based on the maximum chord-length and velocity obtained in the entire data set. Note that, in the case of two-point probes the apparent and actual chord-length and velocity are only the same when the angle of attack between the bubble and the probe is zero. In other cases, four-point probes are required to extract the bubble shape factor and the bubble orientation from probe signals (Lucas and Mishra, 2005; Guet et al., 2005; Luther et al., 2004).

The surface, volume and the most important bubble raw moments, i.e. volume equivalent diameter, surface equivalent diameter, and Sauter diameter, can be obtained as in Eq. (7). The geometrical relationships and the raw moments estimators of such variables are summarized in Table 1.

It is important to notice that in Eqs. (7) (9) the bubble diameter $k$-moment depends on the chord-length $k$ moment, while the surface $k$-moment depends on the chord-length $2 k$-moment and the volume $k$-moment depends on the chord-length $3 k$-moment. Thus, for a limited number of samples, the estimations of the surface and volume moments are not as good as those of the bubble diameter since the deviation of the sampled moments from their real values increases as the order increases. Considering the surface/volume PDFs, their lower moments, which provide the global shape of the distributions, come from the higher order moments of the chord-length. Therefore, to give reliable estimates of moments of the bubble surface and/or volume, it is required a sampling record larger than that needed to estimate the bubble size moments. 
Table 1

Geometrical relationship and raw moments estimators

\begin{tabular}{|c|c|c|c|}
\hline & Geometrical relationship & Raw moments estimator & \\
\hline Surface & $\begin{array}{c}S \pi D^{2} \varepsilon \\
\frac{1}{2}+\frac{\alpha^{2}}{4 \sqrt{ } 1-\alpha^{2}} \ln \left(\frac{1+\sqrt{ } 1-\alpha^{2}}{1-\sqrt{ } 1-\alpha^{2}}\right)\end{array}$ & $\pi^{k} \frac{2 k+2}{2 N} \sum_{i=1}^{N}\left(\frac{\varepsilon_{i}^{1 / 2} y_{i} t_{i}}{\alpha_{i}}\right)^{2 k}$ & (8) \\
\hline Volume & $V \quad \frac{\pi \alpha}{6} D^{3}$ & $\left(\frac{\pi}{6}\right)^{k} \frac{3 k+2}{2 N} \sum_{i=1}^{N}\left(\frac{y_{i} t_{i}}{\alpha_{i}^{2 / 3}}\right)^{3 k}$ & (9) \\
\hline Surface equivalent diameter & $D_{20} \quad\left(\frac{S}{\pi}\right)^{1 / 2}$ & $\frac{k+2}{2 N} \sum_{i}^{N}\left(\frac{\varepsilon_{i}^{1 / 2} y_{i} t_{i}}{\alpha_{i}}\right)^{k}$ & $(10)$ \\
\hline Volume equivalent diameter & $D_{30} \quad\left(\frac{6 V}{\pi}\right)^{1 / 3}$ & $\frac{k+2}{2 N} \sum_{i}^{N}\left(\frac{y_{i} t_{i}}{\alpha_{i}^{2 / 3}}\right)^{k}$ & $(11)$ \\
\hline Volume surface diameter & $D_{32} \quad \frac{6 V}{S}$ & $\frac{k+2}{2 N} \sum_{i}^{N}\left(\frac{y_{i} t_{i}}{\varepsilon_{i}}\right)^{k}$ & (12) \\
\hline
\end{tabular}

Although the method proposed in this paper to estimate the raw moments from the sampled data has been developed for ellipsoidal bubbles, the procedure can be applied to any bubble shape model. In fact, Liu and Clark (1995) obtained the mean and standard deviation for a truncated ellipsoidal bubble.

Though the raw moment estimates are developed here to obtain the MaxEnt density, they can also be used in the parametric method, assuming a PDF (typically Gamma, Rayleigh or lognormal), to obtain the parameters of the PDF (mean and standard deviation for the lognormal, for example). However, we will show that better results can be obtained if a MaxEnt distribution is initially determined, and used later on with the parametric model to extract the shape of the PDFs. Before proceeding any further, we will briefly describe the procedure employed to determine the maximum entropy function (MaxEnt).

\subsection{Maximum entropy (MaxEnt) estimate}

The entropy principle of Shannon yields, for a given set of moments, a density function with the minimum amount of previous information. Jaynes (1957) popularized this method in the field of statistical mechanics and defined the maximum entropy distribution as "uniquely determined as the one which is maximally non-committal with regard to missing information, and that it agrees with what is known, but expresses maximum uncertainty with respect to other matters".

As a result, the problem of estimating the maximum entropy distribution subjected to a given number of known raw-moment constraints can be formulated as follows:

$$
\begin{aligned}
\max _{p(D)} & \int_{D \in \Delta}-p(D) \ln (p(D)) \mathrm{d} D \\
\text { s.t. } & \int_{D \in \Delta} p(D) \mathrm{d} D=1 \\
& \quad \int_{D \in \Delta} D^{i} p(D) \mathrm{d} D=\left\langle D^{i}\right\rangle, \quad i=1, \ldots, K
\end{aligned}
$$

where $p(D)$ is the PDF, $\left\langle D^{i}\right\rangle$ is the $i$ th raw moment, $\Delta$ is the interval variation of the random variable $D$ (the bubble diameter in our case) and the function to maximize is named the Shannon's entropy. 
Ryu (1993) showed that the Lagrangian method produces the following MaxEnt distribution for $K$ moment constraints as a solution of the aforementioned optimization problem:

$$
p_{K}(D, \lambda)=\exp \left(\sum_{i=1}^{K}-\lambda_{i} D^{i}\right) / \int_{D \in \Delta} \exp \left(\sum_{i=1}^{K}-\lambda_{i} D^{i}\right) \mathrm{d} D
$$

where $\lambda_{i}$ is the Lagrange multiplier for the $i$ th moment constraint.

In view of the above result the MaxEnt distribution subjected to known $K$ moment constraints is an exponential series that depends on the Lagrange multipliers for the $K$ moment constraints.

Unfortunately, these results were barely used at a practical level due to difficulties in their numerical implementation until Rockinger and Jondeau (2002) developed a new, stable and fast procedure to find the values of the Lagrange multipliers using the Newton's method. They showed that, if the Hessian matrix is full of rank, the Lagrange multiplier values could be obtained iteratively as

$$
\lambda^{(k)}=\lambda^{(k-1)}-H^{-1}\left(\lambda^{(k-1)}\right) G\left(\lambda^{(k-1)}\right),
$$

starting with $\lambda^{(0)} \quad(0, \ldots, 0)$ and where $H$ and $G$ are the Hessian matrix and the gradient vector respectively, defined as

$$
\begin{aligned}
& H_{i j}\left(\lambda^{(k-1)}\right)=\frac{\partial Q\left(\lambda^{(k-1)}\right)}{\partial \lambda_{i} \partial \lambda_{j}}=\int_{D \in \Delta}\left(D^{i}-\left\langle D^{i}\right\rangle\right)\left(D^{j}-\left\langle D^{j}\right\rangle\right) p_{K}\left(D, \lambda^{(k-1)}\right) \mathrm{d} D \\
& G_{i}\left(\lambda^{(k-1)}\right)=\frac{\partial Q\left(\lambda^{(k-1)}\right)}{\partial \lambda_{i}}=\int_{D \in \Delta}\left(D^{i}-\left\langle D^{i}\right\rangle\right) p_{K}\left(D, \lambda^{(k-1)}\right) \mathrm{d} D
\end{aligned}
$$

Here $Q$ is the potential function to be minimized, given by

$$
Q_{K}(D, \lambda)=\int_{D \in \Delta} \exp \left(\sum_{i=1}^{K}-\lambda_{i}\left(D^{i}-\left\langle D^{i}\right\rangle\right)\right) \mathrm{d} D
$$

For practical purposes, the raw moments are unknown and they must be estimated from the measurements. For example, if a sample of diameters is available, the raw moments of the sample $m_{i}(D)=\frac{1}{N} \sum_{j}^{N}{ }_{1} D_{j}^{i}$ can be used to estimate the raw moments, $\left\langle D^{i}\right\rangle$. Here, $N$ is the sample size and $D_{j}$ the $j$ th diameter measured.

As a result, the MaxEnt distribution, subjected to $K$ moment constraints, can be expressed as

$$
p_{K}(D, \lambda)=\exp \left(\sum_{i=1}^{K}-\lambda_{i}\left(D^{i}-m_{i}(D)\right)\right) / \int_{D \in \Delta} \exp \left(\sum_{i=1}^{K}-\lambda_{i}\left(D^{i}-m_{i}(D)\right)\right) \mathrm{d} D
$$

To calculate the above integrals numerically a 96-point Gaussian quadrature was used, although any other suitable method could be applied.

Note that, the experimental data available from a measuring probe is the chord-length of the bubbles rather than their diameter. Therefore, the moments of the sampled diameter (for example) cannot be used to estimate the raw moments, $\left\langle D^{i}\right\rangle$, and a function of the known variables (chord, shape and angle) must be used to determine them (see Eq. (7)).

Thus, if an estimator for raw moments of the bubble diameter, area or volume is available, the MaxEnt distribution of bubble diameter, area or volume can be efficiently determined. Notice that the procedure can be performed avoiding the use of the backward transformation previously proposed by Liu and Clark (1995) for bubbles with a purely vertical rise and revised later on by Santana and Macías-Machín (2000) for bubbles with a non-vertical rise.

Of course, the bubble surface density function can be obtained using the same procedure described by Eqs. (1) (18) for the bubble size PDF but with the bubble surface raw moments obtained from the experimental measurements with Eq. (8), or using the MaxEnt estimate for the surface equivalent diameter as follows:

$$
p_{K}(S, \lambda)=\frac{\exp \left(\sum_{i 0}^{K}-\lambda_{i}\left(\frac{S}{\pi}\right)^{i / 2}\right)}{2 \pi\left(\frac{S}{\pi}\right)^{1 / 2}}
$$


Similarly, the bubble volume density function can also be deduced from the volume equivalent diameter MaxEnt estimate as

$$
p_{K}(V, \lambda)=\frac{\exp \left(\sum_{i 0}^{K}-\lambda_{i}\left(\frac{6 V}{\pi}\right)^{i / 3}\right)}{\frac{\pi}{2}\left(\frac{6 V}{\pi}\right)^{2 / 3}}
$$

This last procedure, given by Eqs. (20) and (21), has been shown to be more precise to deduce the surface or volume distributions than the MaxEnt distribution obtained from the moments in Eqs. (8) and (9) as will be shown in Fig. 9.

\section{Experimental and simulation procedure}

To clarify the procedure described in the previous section, we will determine the size, surface and volume PDFs of bubbles injected in a water tank. Since we did not have access to crossing probe to perform our measurements, the bubble diameter, shape factor and angle of attack was obtained by image processing of images acquired with a high-speed camera, and the chord-lengths were synthetically generated with a Monte-Carlo method. Thus, a cloud of rising bubbles was generated by injecting a certain flow of air through a hypodermic needle located at the bottom of an acrylic tank filled with water. The dimensions of the tank, $500 \mathrm{~mm} \times 500 \mathrm{~mm}$ of cross-sectional area (Fig. 2), were large enough to ensure that the side walls did not affect the motion of the air bubbles. In addition, in all the experiments reported here the measuring window was placed $100 \mathrm{~mm}$ downstream of the injection point and more than $500 \mathrm{~mm}$ below the free surface to avoid undesired effects of either the bottom or the free surface.

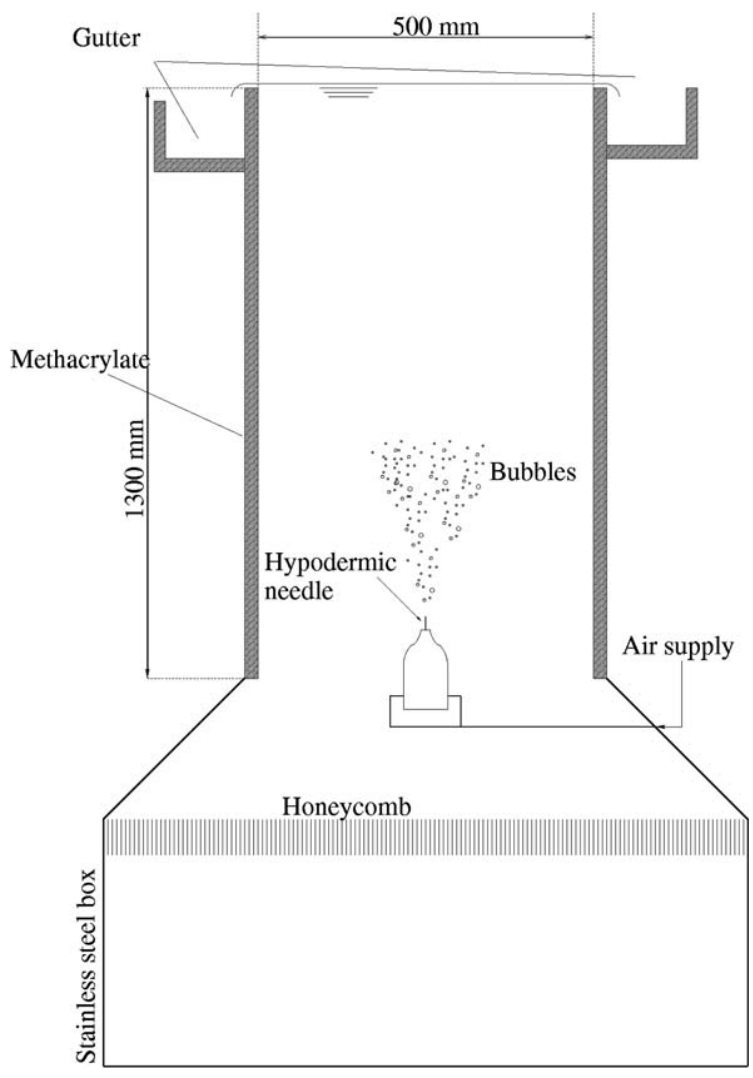

Fig. 2. Experimental set up. 
The time evolution of the bubbles, as they were rising up, was recorded with a Kodak Ektrapro high-speed camera, working at 1000 frames per second. At the working frame rate, the resolution of the images was $256 \times 240$ pixels, which corresponded to a measuring window of $16 \mathrm{~mm} \times 15 \mathrm{~mm}$. The position of the bubbles was obtained using the tracking algorithm described in Rodríguez-Rodríguez et al. (2003). Since this algorithm was developed to detect the breakage or coalescence of bubbles, it was also able to detect overlapping bubbles within the images. Therefore, when a cluster of two or more overlapping bubbles was found, the algorithm automatically rejected it avoiding the acquisition of undesired data.

In each frame of the high-speed movie, the coordinates of the centroid of any single bubble that crossed the virtual probe was determined and employed to compute its bubble instantaneous velocity, the minor and major axes $(D, \alpha D)$ of the ellipse that best fitted the perimeter of the bubble, as well as the angle between the minor axis and the velocity vector, $\theta$. An illustrative example is shown in Fig. 3. A sample of 350 bubbles was obtained experimentally. This procedure provides a $2 \mathrm{D}$ projection of a $3 \mathrm{D}$ bubble and, consequently, when the projection corresponds to the side view rather than to the front view, the shape factor may be overestimated by a factor as shows Fig. 4. Therefore, assuming that bubble inclination with respect to the vertical direction is isotropic, the errors in the characteristic lengths have been obtained and given in Table 2.

Unfortunately, it was not possible to extract the chord-length of the bubbles crossing the virtual probe, $y$, from the two-dimensional images. Thus, several sets of chord-lengths were synthetically generated for the ellipsoidal bubble model with the Monte-Carlo method, using the experimental values of $D, \alpha, \theta$, obtained from the images processed, and applying Eq. (1). An example of five different simulations of chord-length samples obtained from the same 350 bubbles recorded in a characteristic data set is showed in Fig. 5 .

The bubble size, area or volume moments could be estimated for each set of chord-lengths generated and, consequently, their MaxEnt distributions could be calculated as follows:

(1) First the domain $\Delta$ where the diameters $\left(D, D_{20}, D_{30}, D_{32}\right)$, area $(S)$ or volume $(V)$ PDFs was selected. For our simulations, the lower bound was set to 0 and the upper one was taken slightly larger than the maximum bubble size, area or volume obtained from the chord-lengths simulated.

(2) Second, the MaxEnt distribution was estimated subjected to $K$ known sample moment constraints. In our case we started with $K \quad 2$, and used Eq. (15) with the initial values $\lambda^{(0)}=(0, \ldots ., 0)$, until the convergence criterion $G\left(\lambda^{(k 1)}\right) \leqslant 10^{6}$ was fulfilled. It must be said that procedure normally reached the convergence criteria in less than 10 iterations.

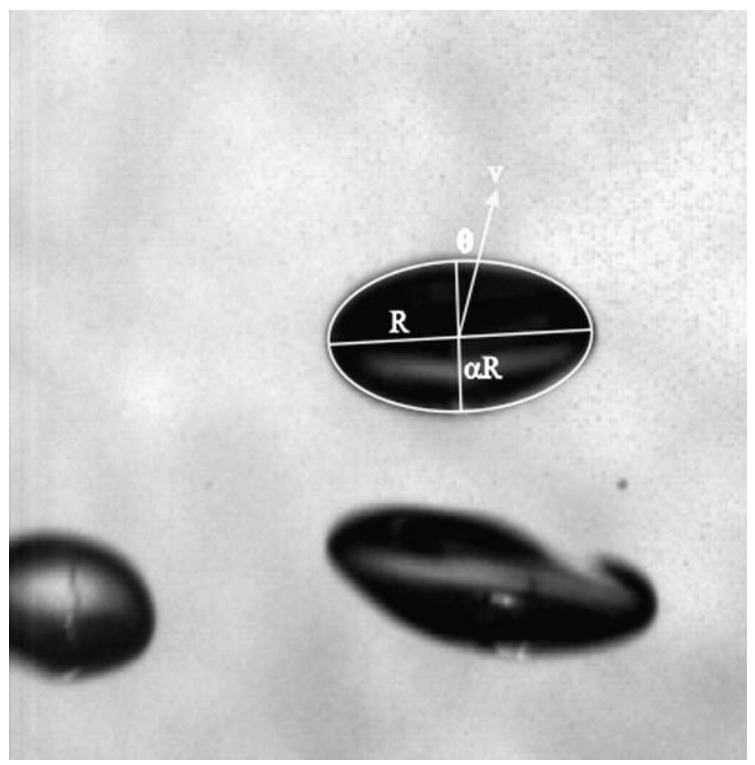

Fig. 3. Image of a bubble together with its best fit ellipse. 


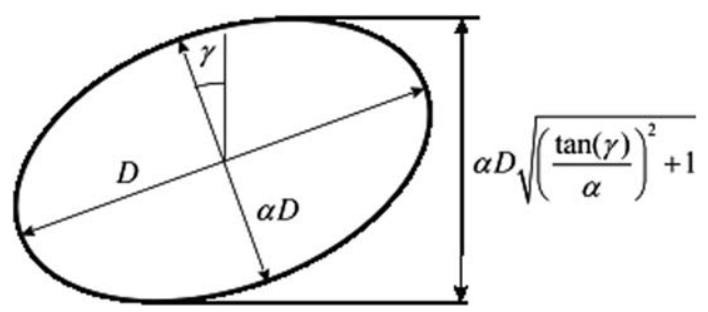

Fig. 4. Measured minor semi axis of an oblate spheroid from image analysis (lateral view).

Table 2

Variables overestimation from $2 \mathrm{D}$ bubble projections

\begin{tabular}{lllllll}
\hline Variable & $\alpha$ & $D_{20}$ & $D_{30}$ & $D_{32}$ & $S$ & $V$ \\
\hline Relative error $(\%)$ & 15 & 3.5 & 4.8 & 7.3 & 7.3 & 15 \\
\hline
\end{tabular}

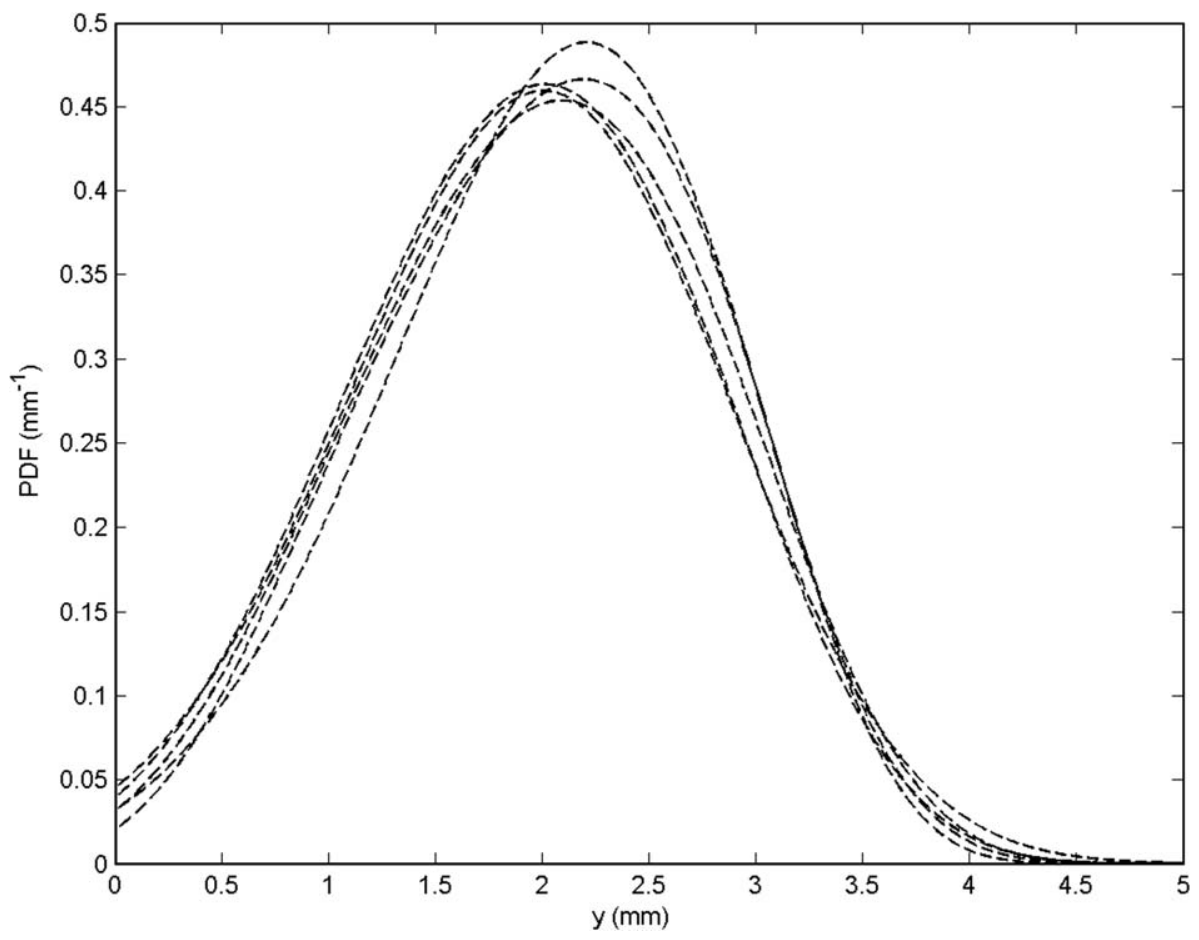

Fig. 5. Synthetically generated chord length distributions from the two dimensional images.

(3) Finally, steps (1) and (2) were repeated until the Hessian matrix of Eq. (15) was not full of rank, or K 10 .

Notice that this process is very easy to implement and has a very low computational cost. The procedure also allows us to calculate a maximum of nine MaxEnt distributions, fixing a maximum of 10 raw sample moments.

However, since the real moments are unknown and the error of the raw moment estimates increases as their order increases, we have to decide which one of the $K$ different PDF calculated best fits the true PDF. For that purpose, the Kullback Leibler entropy discrepancy criterion (Kullback and Leibler, 1951) was applied. This criterion indicates that if the Kullback Leibler discrepancy, $\Delta_{K L}$, given by 


$$
\Delta_{K L}(K)=\int_{\Delta} p_{K}(D) \log \left(p_{K}(D) / p_{K+1}(D)\right) \mathrm{d} D
$$

is minimum near 0 , the two distributions are the same and the inclusion of a new moment constraint does not contribute to maximize the information content of the PDF. Thus, the optimum distribution must be the MaxEnt distribution that fixes the maximum number of moment constraints $(K)$ and has the minimum Kullback Leibler discrepancy value.

To clarify the different concepts above described, we will apply our semi-parametric procedure to our experimental measurements with the aim at determining the best-fitted bubble size distribution in the following section.

\section{Results and discussion}

Fig. 6 shows the MaxEnt distributions calculated from the experimental measurements above reported for an increasing number of the moment constrains, together with the one experimentally measured. It can be observed that the MaxEnt distribution becomes closer to the experimental one as the number of moment constrains increases from $K \quad 2$ to $K \quad 5$. The Kullback Leibler entropy discrepancy criterion, also shown in Fig. 6 as an inset, decreases with the number of moment constraints until the MaxEnt distribution is subjected to the 6th raw sample moment. Therefore, for this simulation, the optimum MaxEnt distribution is the one that fixes the first five raw sample moments and, the inclusion of the 6th raw sample moment does not improve the estimated distribution.

Fig. 7 shows the comparison of the optimal MaxEnt distributions (dashed lines), obtained from the five simulations of the chord-length showed in Fig. 5, together with the experimental distribution (solid lines) of four of the most important characteristic diameters, namely $D, D_{20}, D_{30}, D_{32}$. To obtain the optimal MaxEnt distributions of $D_{20}, D_{30}, D_{32}$ we followed the same procedure described to obtain MaxEnt distribution of $D$ but using the experimental values of $D_{20}, D_{30}$, and $D_{32}$ for each of the 350 bubbles recorded in our measurements.

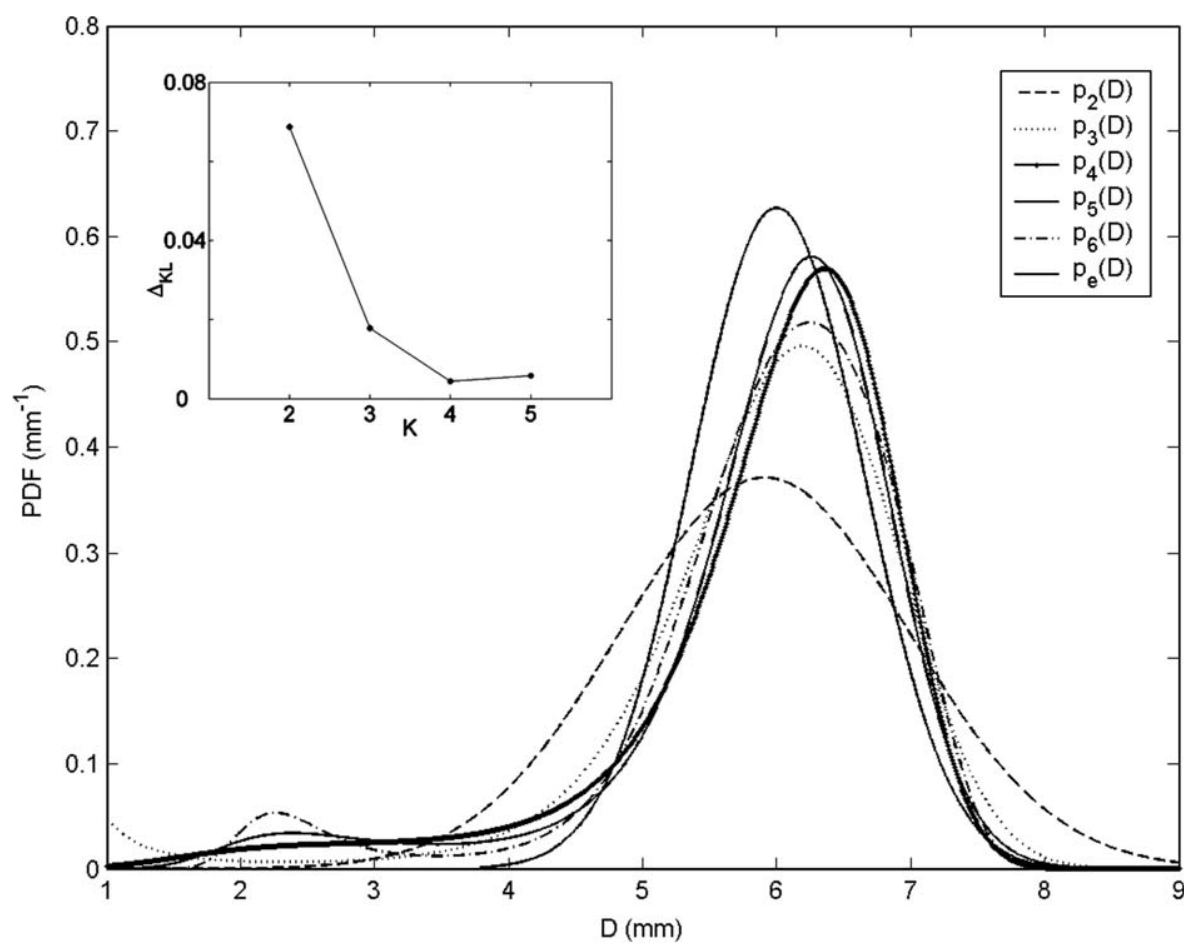

Fig. 6. Effect of the number of moment constraints on the MaxEnt estimation. 

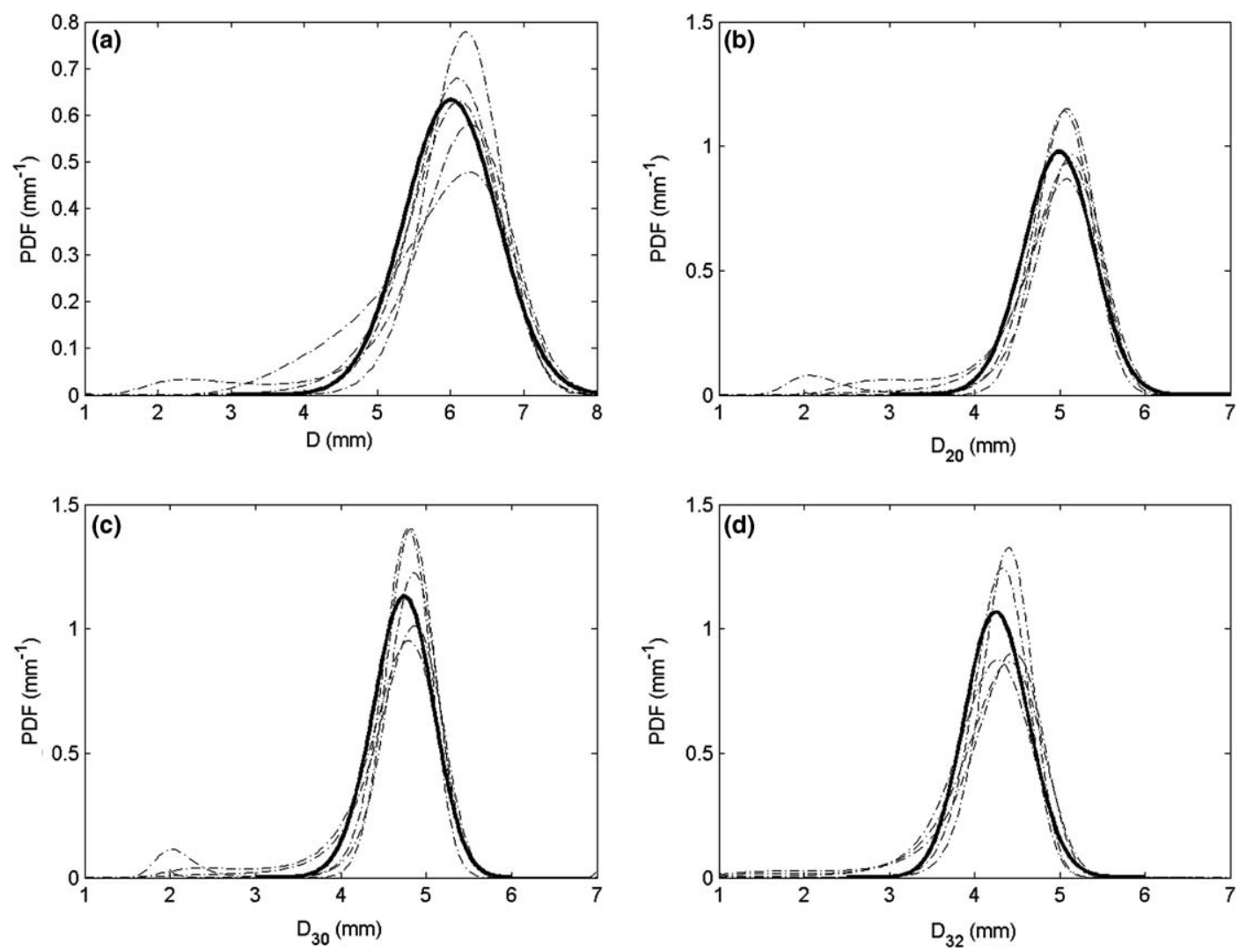

Fig. 7. Optimal MaxEnt estimation obtained from the five simulations of the chord length showed in Fig. 5, together with the experimental distribution (solid lines), for (a) bubble diameter, $D$, (b) surface equivalent diameter, $D_{20}$, (c) volume equivalent diameter, $D_{30}$, (d) Sauter mean diameter for five simulations, $D_{32}$.

It can be observed in Fig. 7 that the MaxEnt estimation becomes very close to the experimental distribution for all the characteristics diameters. Note that for these approximations, a record of only 350 bubbles were used, whereas other non-parametric methods, i.e. Liu et al. $(1996,1998)$ and Santana and Macías-Machín (2000), require almost 5000 bubbles and need to use a backward transformation to obtain a reliable distribution. Consider that, if the bubble shape cannot be obtained experimentally, it must be inferred from the bubble velocity, for example, and, consequently, the error in the calculations of the raw sampled moments would increase due to the uncertainty in the bubble shape estimation.

Fig. 8 shows the bias of the first sample moments of the above mentioned characteristic diameters, namely mean, standard deviation, skewness and kurtosis, with respect to the experimental ones (indicated with a solid circle). Notice that as the order of the moment increases, $K$, its deviation from the experimental value also increases, being the kurtosis the one showing the largest deviations. However this bias could be reduced increasing the number of bubbles in the sample. The definition of the first moments, together with the mean values of the first four moments of the experimental and simulated characteristic diameters showed in Fig. 8 is provided in Table 3 for comparison purposes.

Fig. 9 shows the bubble surface and the volume PDFs estimated from the optimal MaxEnt distribution calculated from Eqs. (14) (18) for $S$ and $V$, using the surface and volume sampled raw moments given by Eqs. (8) and (9) respectively (Fig. 9a and c). In addition, Fig. 9 also shows the bubble surface and volume PDFs estimated from Eqs. (20) and (21) using the Lagrange multipliers, $\lambda_{i}$, corresponding to $D_{20}$ and $D_{30}$ respectively (Fig. 9b and d). The different curves shown in each plot represent the distributions obtained from five different Monte-Carlo simulations of the bubble chord-length. It can be seen that the PDFs given by Eqs. (20) and (21) estimate the real PDF slightly better than the optimal MaxEnt estimation. Perhaps the most important 


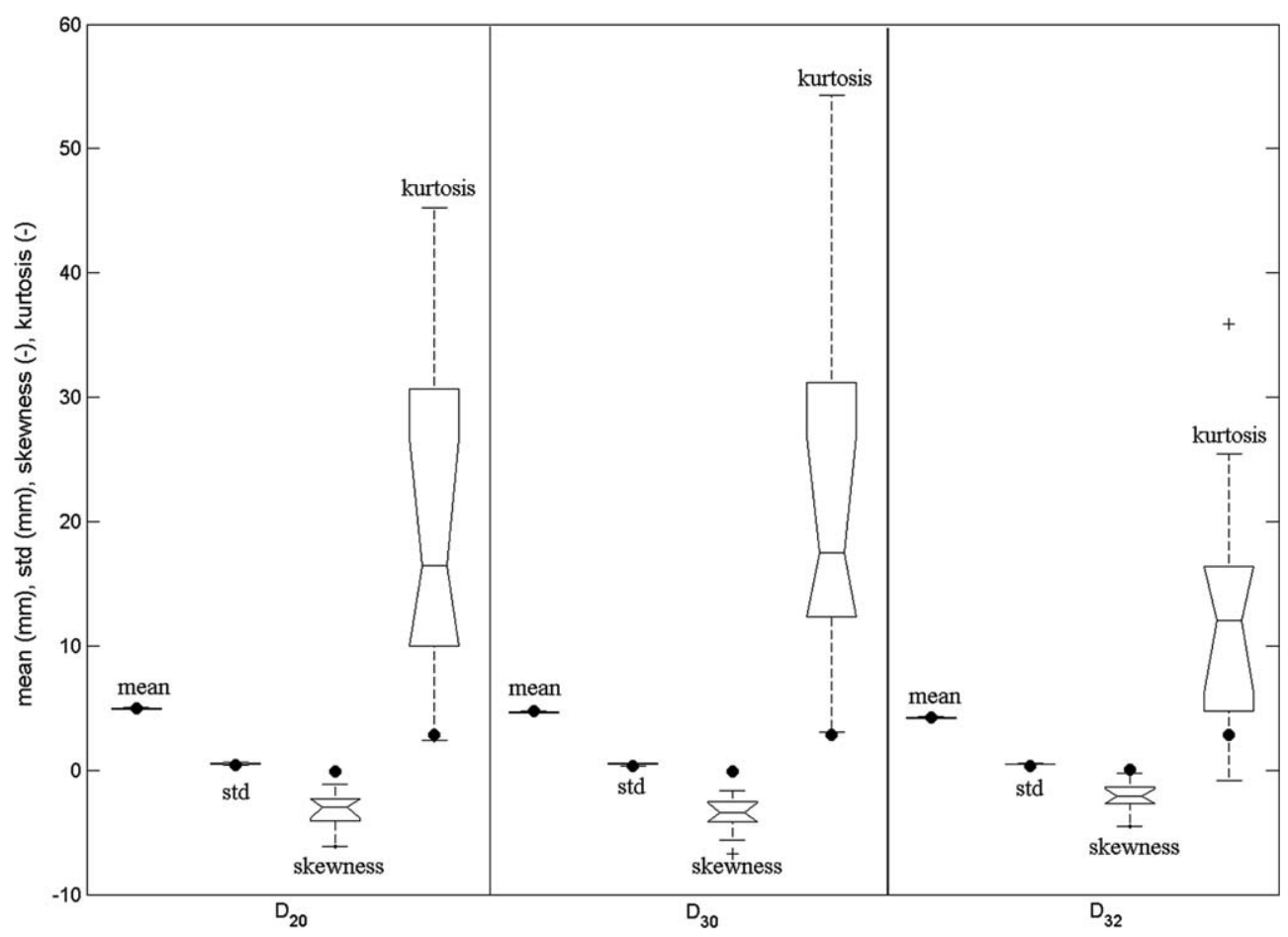

Fig. 8. Deviation of the first sample moments, i.e. mean, standard deviation, skewness and kurtosis, of three characteristics diameters $\left(D_{20}, D_{30}, D_{32}\right)$ with respect to the experimental values.

Table 3

Error in the calculation of the first four moments of the characteristic diameters shown in Fig. 8

\begin{tabular}{|c|c|c|c|c|c|}
\hline & & Mean (mm) & Std $(\mathrm{mm})$ & Skewness ( ) & Kurtosis ( ) \\
\hline & & $\mu=\langle x\rangle$ & $\left.\sigma \quad \sqrt{\langle(x} \quad \mu)^{2}\right\rangle$ & $\frac{\left\langle(x-\mu)^{3}\right\rangle}{\sigma^{3}}$ & $\frac{\left\langle(x-\mu)^{4}\right\rangle}{\sigma^{4}}$ \\
\hline \multirow[t]{2}{*}{$D_{20}$} & Experimental & 4.98 & 0.41 & 0.08 & 2.82 \\
\hline & Simulated & 4.92 & 0.54 & 3.29 & 19.73 \\
\hline \multirow[t]{2}{*}{$D_{30}$} & Experimental & 4.72 & 0.35 & 0.11 & 2.83 \\
\hline & Simulated & 4.67 & 0.49 & 3.62 & 22 \\
\hline \multirow[t]{2}{*}{$D_{32}$} & Experimental & 4.26 & 0.37 & 0.08 & 2.85 \\
\hline & Simulated & 4.22 & 0.47 & 2.24 & 13.46 \\
\hline
\end{tabular}

advantage of the use of Eqs. (20) and (21) to estimate the bubble surface and bubble volume PDFs is that the probability to obtain bubbles of zero surface or volume is zero (see Fig. $9 \mathrm{~b}$ and c). Thus, the bubble surface and volume PDFs are strictly positive.

As previously mentioned, Fig. 10, which shows the deviation of the first sample moments, i.e. mean, standard deviation, skewness and kurtosis, of the bubble surface and bubble volume with respect to their experimental values, indicates that the surface $k$-moment depends on the chord-length $2 k$-moment and the volume $k$-moment depends on the chord-length $3 k$-moment. Consequently, the surface and volume moments, especially the mean and the standard deviation, are not as well estimated as their corresponding values for the bubble diameters (shown in Fig. 8) for a finite number of samples since the sampled moments deviate from the true moments as $k$ increases. This result can be corroborated comparing the errors extracted from Tables 3 and 4 respectively. The low moments of the surface or volume PDFs, that provide its global shape, come from the higher order chord-length moments and, therefore, in order to give a reliable estimate of moments of the bubble surface or volume, a bubble sampling record larger than that needed for the estimate of bubble size moments is required. 

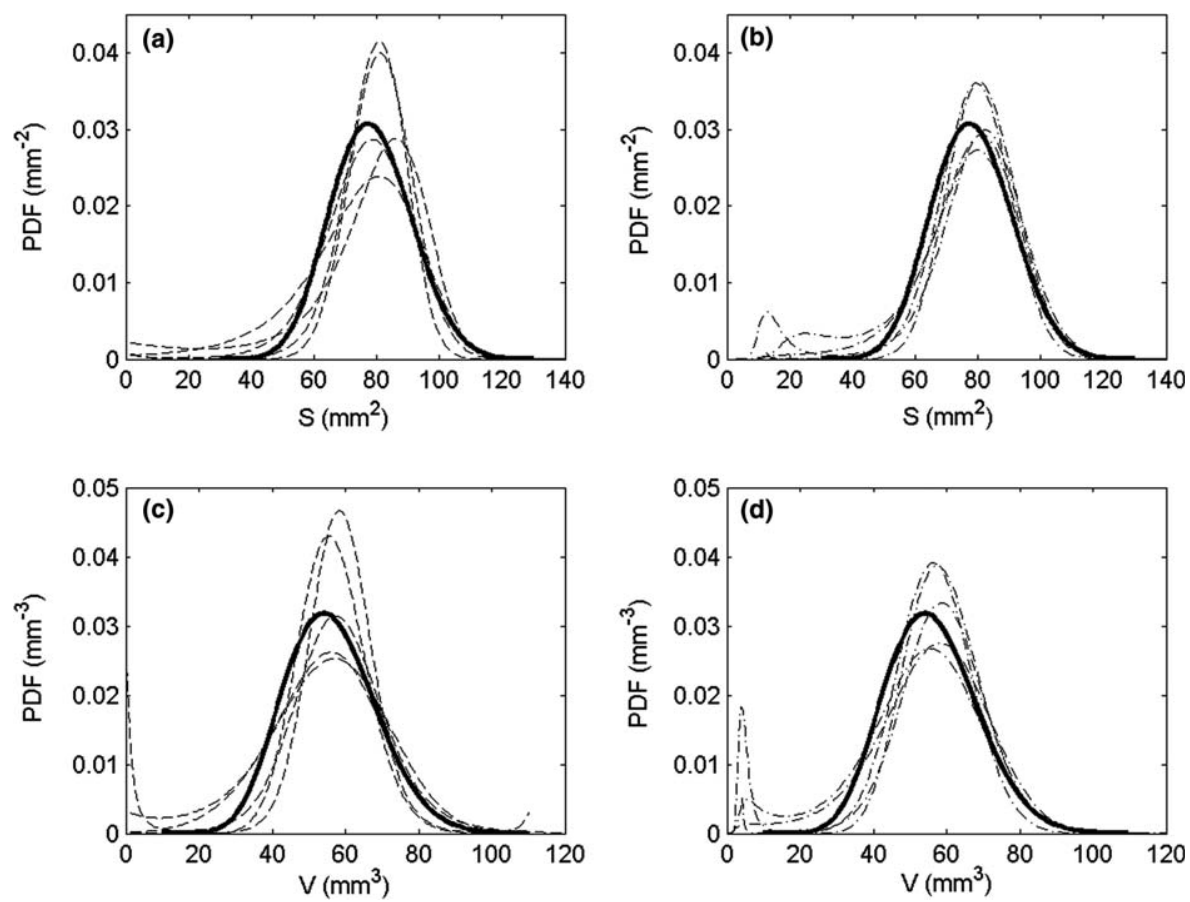

Fig. 9. (a) Optimal MaxEnt estimation for bubble surface, (b) optimal estimation for bubble surface from Eq. (20), (c) optimal MaxEnt estimation for bubble volume, (d) optimal estimation for bubble volume from Eq. (21).

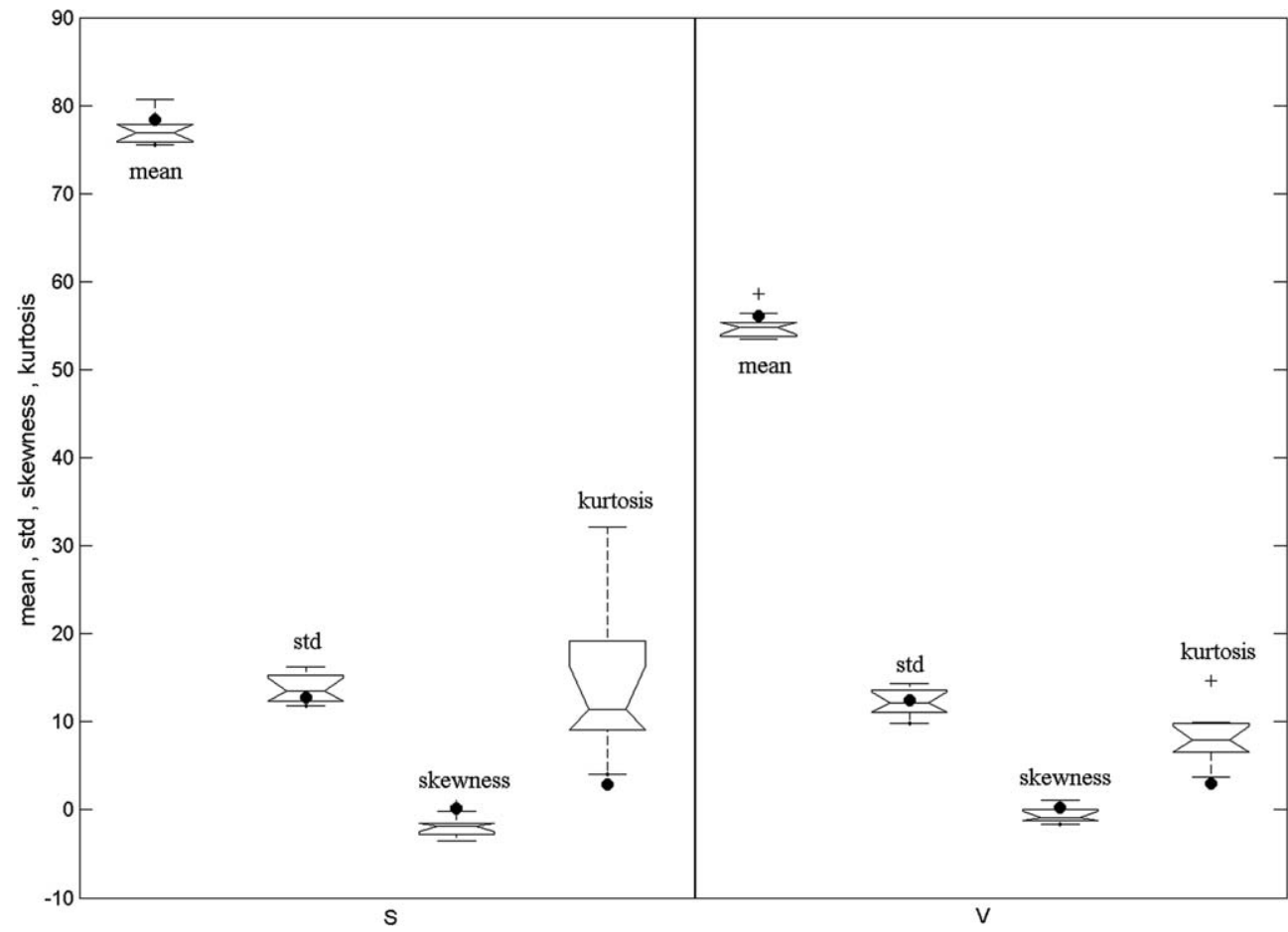

Fig. 10. Deviation of the first sample moments, i.e. mean, standard deviation, skewness and kurtosis, of the bubble surface and bubble volume with respect to the experimental values. 
Obviously as the sample size increases, a better distribution is obtained, and a more reliable PDF can be achieved from the experimental data. Thus, Fig. 11 shows that the MaxEnt distribution, obtained for one of the sequences of chord-length simulated, approximates better to the real one as the number of samples increases from 50 to 350 . This figure also shows that error in the mean value of the distribution scarcely changes when the number of samples is larger than 150 in this case. Similarly, as the sample size increases the other higher-order moments approach to their respective experimental value. The convergence of these moments to the experimental values is initially very fast as the sample size increase, indicating that the sample size can be reduced drastically compared to the non-parametric approaches proposed by Liu et al. $(1996,1998)$ and Santana and Macías-Machín (2000).

Since, shape and velocity of spiralling ellipsoidal bubbles are difficult to accurately measure with sampling probes, it seems reasonable to briefly discuss the dependence of the results with the accuracy of the measurements. Thus as can be deduced from Eq. (6), the errors in the bubble diameter estimation are directly propor-

Table 4

Error in the calculation of the first four moments of the bubble surface and volume shown in Fig. 10

\begin{tabular}{|c|c|c|c|c|}
\hline & \multicolumn{4}{|l|}{$S$} \\
\hline & $\operatorname{Mean}\left(\mathrm{mm}^{2}\right)$ & Std $\left(\mathrm{mm}^{2}\right)$ & Skewness ( ) & Kurtosis ( ) \\
\hline Experimental & 78.34 & 12.78 & 0.14 & 2.87 \\
\hline \multirow[t]{3}{*}{ Simulated } & 77.18 & 13.83 & 1.87 & 14.64 \\
\hline & \multicolumn{4}{|l|}{$V$} \\
\hline & Mean $\left(\mathrm{mm}^{3}\right)$ & Std $\left(\mathrm{mm}^{3}\right)$ & Skewness ( ) & Kurtosis ( ) \\
\hline Experimental & 56.11 & 12.45 & 0.3 & 3.01 \\
\hline Simulated & 55 & 12.21 & 0.55 & 8.12 \\
\hline
\end{tabular}

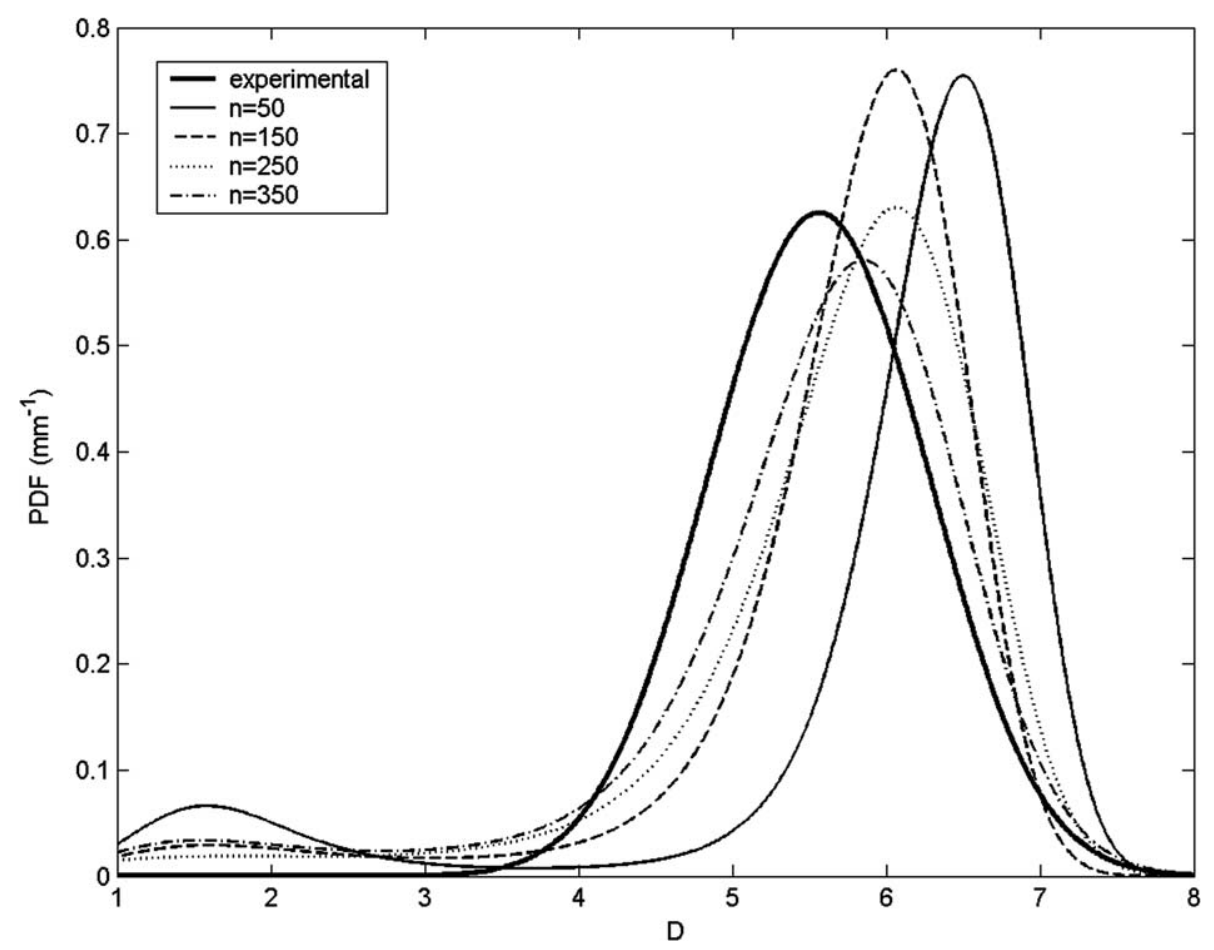

Fig. 11. Evolution of the MaxEnt distribution with the number of samples. 
tional to the errors in the measurements of the chord-length. Moreover, an overestimation (underestimation) of the mean or standard deviation of the chord-length produces the same overestimation (underestimation) of the mean and standard deviation of the bubble diameter. On the other hand, the overestimation (underestimation) of the mean or standard deviation of the shape factor is inversely proportional to the overestimation (underestimation) of the mean and standard deviation of the bubble diameter. The errors in the angle of attack (errors in term $t$ in Eq. (6)) do not affect appreciably the diameter estimation. These results can be observed in Fig. 12, where it has been represented the effect of noise addition to each of the measurable, or estimated variables $(y, \alpha, \theta)$ from the probe signals on the MaxEnt distribution. The artificial noise added, consists of uniform noise of zero mean value and maximum relative error $E$. For example Fig. 12(a) shows a comparison between the MaxEnt distributions obtained for distribution of chord-lengths, $y_{i}$, and the same one with noise addition, $y_{\mathrm{er}, i} \quad y_{i}(1+E)$, where $E$ is a uniform distribution ranging from $-E$ to $E$. As Fig. 12 indicates, the most important variables to obtain a reliable PDF are the chord-length and the shape factor. This figure also shows that the errors in the angle of attack produce a negligible effect on the PDF estimation (Fig. 12(c)). Similar results have been found in the estimation of the other characteristic diameters, $D_{20}, D_{30}, D_{32}$. Furthermore, Fig. 13 shows the effect of inaccurate measurements of the bubble shape factor on $D_{20}, D_{30}, D_{32}$. It can be seen that noise addition affects in great extent to the surface and volume equivalent diameter distributions, whereas its effect on the Sauter diameter distribution is somehow weaker due to the dependence of each characteristic diameter on the shape factor as indicated in Table 1 . Notice that, $D_{20}$ depends on $\alpha$ as approximately $\alpha^{3 / 4}$, while $D_{32}$ depends as $\alpha^{1 / 2}$. This dependence produces a worse estimation in the probability distribution of $D_{20}$ than that of $D_{32}$ when the same error is made in the shape factor estimation or measurement.
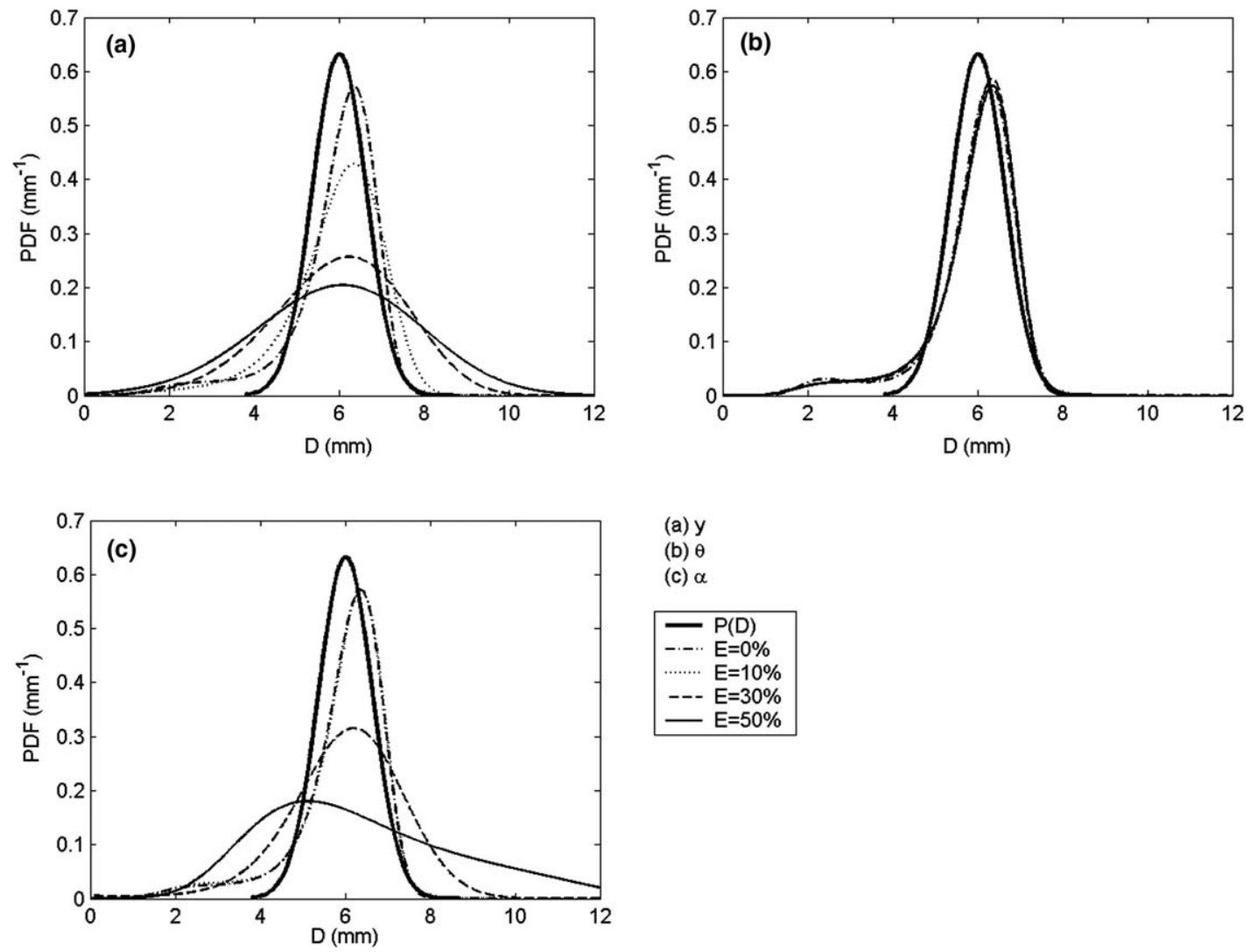

Fig. 12. Effect of noise addition on the estimation of bubble diameter PDF. The noise has been added to the input data of (a) chord length, (b) angle of attack and (c) shape factor. 

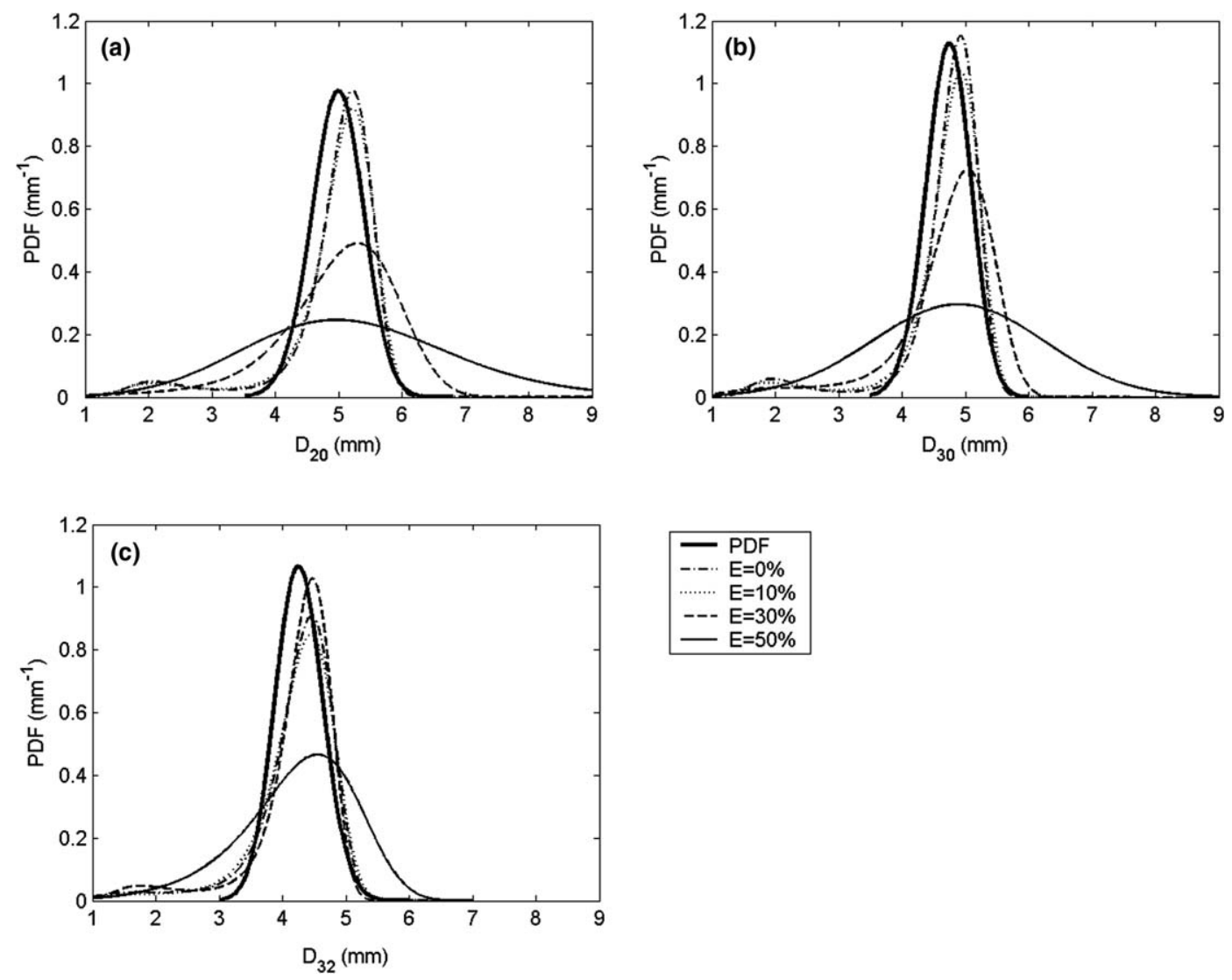

Fig. 13. Effect of noise addition to shape factor on the estimation of bubble characteristic diameter PDFs: (a) $D_{20}$, (b) $D_{30}$ and (c) $D_{32}$.

\section{Conclusion}

A stable and fast procedure to estimate the bubble size, surface and volume distributions from sample moments obtained from probe signals are proposed. The raw moments estimated are restricted to the ellipsoidal bubble regime.

The most important length scales in two-phase flow: diameter, surface equivalent diameter, volume equivalent diameter and Sauter diameter can be obtained from probe signals.

Furthermore, the maximum entropy (MaxEnt) estimated distribution fixes almost five sample moments, avoiding the use of a backward transform. Additionally it is free of constant shape factor assumption.

The sample needed to obtain reliable distributions using the maximum entropy distribution is generally less than the sample needed in non-parametric methods using backward transform.

The method proposed is optimal to infer bubble size distribution from probe signals and promising for using it on population balance model, as only bubble sample moments are needed to infer bubble size, surface or volume distributions.

\section{Acknowledgement}

This work has been supported by the Spanish Ministry of Education and European Union under project number DPI2005-08654-C04-01. 


\section{References}

Boyer, C., Duquenne, A.M., Wild, G., 2002. Measuring techniques in gas liquid and gas liquid solid reactors. Chem. Eng. Sci. 57, 3185 3215.

Cartellier, A., 1999. Post treatment for phase detection probes in non uniform two phase flows. Int. J. Multiphase Flow 25, 201228.

Chaumat, H., Billet Duquenne, A.M., Augier, F., Mathieu, C., Delmas, H., 2005. Application of the double optic probe technique to distorted tumbling bubbles in aqueous or organic liquid. Chem. Eng. Sci. 60, 61346145.

Cheremisinoff, N.P., 1986. Review of experimental methods for studying the hydrodynamics of gas solid fluidized beds. industrial and engineering chemistry. Process Des. Dev. 25, 329351.

Clark, N.N., Turton, R., 1988. Chord length distributions related to bubble size distributions in multiphase flows. Int. J. Multiphase Flow $14,413424$.

Clark, N.N., Liu, W.D., Turton, R., 1996. Data interpretation techniques for inferring bubble size distribution from probe signals in fluidized systems. Powder Technol. 88, 179188.

Clift, R., Grace, J.R., Weber, M.E., 1978. Bubbles, Drops and Particles. Academic Press, New York.

Dias, S.G., França, F.A., Rosa, E.S., 2000. Statistical method to calculate local interfacial variables in two phase bubbly flows using intrusive crossing probes. Int. J. Multiphase Flow 26, 17971830.

Guet, S., Luther, S., Ooms, G., 2005. Bubble shape and orientation determination with a four point. Exp. Therm. Fluid Sci. $29,803812$.

Herringe, R.A., Davis, M.R., 1976. Structural development of gas liquid mixture flows. J. Fluid Mech. 73, 97123.

Jaynes, E.T., 1957. Information theory and statistical mechanics. Phys. Rev. 106, 620630.

Julia, J.E., Harteveld, W.K., Mudde, R.F., Van den Akker, H.E.A., 2005. On the accuracy of the void fraction measurements using optical probes in bubbly flows. Rev. Sci. Instrum. 76, 035103113.

Kalkach Navarro, S., Lahey, R.T., Drew, D.A., Meyer, R., 1993. Interfacial area density, mean radius and number density measurements in bubbly 2 phase flow. Nucl. Eng. Des. 142, 341351.

Karamanev, D.G., 1994. Rise of gas bubbles in quiescent liquids. AIChE J. 40, 14181421.

Kullback, S., Leibler, R.A., 1951. On information and sufficiency. Ann. Math. Stat. 22, 7986.

Lee, S.L.P., Soria, A., Lasa, H., 1990. Evolution of bubble length distribution in 3 phase fluidized beds. AIChE J. 40 , 17631767.

Liu, W., Clark, N.N., 1995. Relationships between distributions of chord lengths and distributions of bubble size including their statistical parameters. Int. J. Multiphase Flow 21, 10731089.

Liu, W.D., Clark, N.N., Karamavruc, A.I., 1996. General method for the transformation of chord length data to a local bubble size distribution. AIChE J. 42, 27132720.

Liu, W.D., Clark, N.N., Karamavruc, A.I., 1998. Relationship between bubble size distribution and chord length distribution in heterogeneously bubbling systems. Chem. Eng. Sci. 53, 12671276.

Lucas, G.P., Mishra, R., 2005. Measurement of bubble velocity components in a swirling gas liquid pipe flow using a local four sensor conductance probe. Meas. Sci. Technol. 16, 749758.

Luther, S., Rensen, J., Guet, S., 2004. Bubble aspect ratio and velocity measurement using a four point fiber optical probe. Exp. Fluids 36, 326333.

Mudde, R.F., Saito, T., 2001. Hydrodynamical similarities between bubble column and bubbly pipe flow. J. Fluid Mech. $437,203228$.

Nguyen, A.V., 1998. Prediction of bubble terminal velocities in contaminated water. AIChE J. 44, 226230.

Rockinger, M., Jondeau, E., 2002. Entropy densities with an application to autoregressive conditional skewness and kurtosis. J. Econ. 106, 119142.

Rodríguez Rodríguez, J., Martínez Bazán, C., Montanes, J.L., 2003. A novel particle tracking and break up detection algorithm: application to the turbulent break up of bubbles. Meas. Sci. Technol. 14, 13281340.

Ryu, H.K., 1993. Maximum entropy estimation of density and regression functions. J. Econ. 95, 131440.

Santana, D., Macías Machín, A., 2000. Local bubble size distribution in fluidized beds. AIChE J. 46, 13401347.

Taylor, T.D., Acrivos, A., 1964. On the deformation and drag of a falling viscous drop at low Reynolds number. J. Fluid Mech. 18,466 476.

Turton, R., Clark, N.N., 1989. Interpreting probe signals from fluidized beds. Powder Technol. 59, 117123.

Wellek, R.M., Agrawal, A.K., Skelland, A.H., 1966. Shape of liquid drops moving in liquid media. AICHE J. $12,854862$.

Werther, J., 1974. Bubbles in gas fluidised beds Part I. Trans. Inst. Chem. Eng. 52, 149159. 